\title{
Multilinguales
}

1 | 2013

Pratiques littéraires, linguistiques, pédagogiques,

didactiques et médiations culturelles contemporaines

\section{De la notion de « langue appliquée » : vers une nécessaire autonomie}

The notion of applied language: towards a necessary autonomy

\section{M'hand Ammouden}

\section{(2) OpenEdition}

\section{Journals}

Édition électronique

URL : https://journals.openedition.org/multilinguales/3012

DOI : $10.4000 /$ multilinguales.3012

ISSN : 2335-1853

Éditeur

Université Abderrahmane Mira - Bejaia

Édition imprimée

Date de publication : 1 juin 2013

Pagination : 59-68

ISSN : 2335-1535

\section{Référence électronique}

M'hand Ammouden, « De la notion de « langue appliquée » : vers une nécessaire autonomie », Multilinguales [En ligne], 1 | 2013, mis en ligne le 01 juin 2013, consulté le 30 juin 2021. URL : http:// journals.openedition.org/multilinguales/3012; DOI : https://doi.org/10.4000/multilinguales.3012

Ce document a été généré automatiquement le 30 juin 2021.

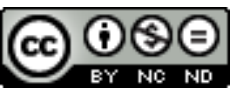

Multilinguales est mise à disposition selon les termes de la Licence Creative Commons Attribution -

Pas d'Utilisation Commerciale - Pas de Modification 4.0 International 


\title{
De la notion de « langue appliquée » : vers une nécessaire autonomie
}

The notion of applied language: towards a necessary autonomy

\author{
M'hand Ammouden
}

1 Les cursus universitaires intègrent de plus en plus des formations en langues appliquées (désormais LA) et ce, dans plusieurs pays, dont l'Algérie. Leur place prépondérante nous autorise à parler d'émergence d'un nouveau champ de réflexion. Cependant, dans les discours qui traitent de ces formations, celles-ci, en général, ne sont pas considérées comme constituant un champ autonome. Quand elles ne sont pas simplement placées dans le vaste champ des langues étrangères en tant que « langues étrangères appliquées" (désormais LEA), elles sont souvent présentées comme appartenant à des sous-champs de la didactique des langues dont les formations sont destinées à des publics spécifiques ${ }^{1}$ (désormais PS). En général, les LA sont assimilées à des langues de spécialité (désormais LS) ou à des langues sur objectifs spécifiques (désormais LOS).

2 Cet amalgame a des conséquences sur le plan didactique, puisqu'on associe aux formations en LA des logiques d'enseignement/apprentissage qui ne leur correspondent pas ou peu, réduisant ainsi leurs velléités de développement dans leur spécificité.

3 Le nombre de plus en plus important d'offres de formation en LA, le caractère (très) nouveau ${ }^{2}$ de ces profils de formation, le fait que les LA ne soient pas encore (suffisamment) théorisées, comparées aux LS ou aux LOS, nous ont amené à réfléchir au problème que pose la terminologie "langue appliquée». Cette contribution en est le premier jalon. Nous nous référerons essentiellement à l'expression elle-même «langue appliquée » - et à des descriptifs de formations initiées dans ce cadre. Cette réflexion nous permettra de nous interroger d'abord sur l'affiliation des formations en LA au champ de la didactique des PS, puis, sur la validité de l'insertion de ces 
formations dans les champs des LS ou des LOS, et enfin, sur la possibilité qu'elles ont de constituer un nouveau sous-champ de la didactique des langues.

\section{Des langues destinées à des publics spécifiques ?}

4 Les LA sont-elles destinées aux publics des langues dites «communes" ou " ordinaires ", ou sont-elles réservées à des publics spécifiques ? Pour répondre à cette question, il nous faut confronter les caractéristiques des formations en LA avec celles des formations appartenant au champ de la didactique des PS.

5 L'expression générique " public spécifique » est, en principe, utilisée pour désigner les publics concernés par des formations en langues dans une spécialité donnée. Celles-ci sont à distinguer de celles en langues « communes ». Elle est, par exemple, utilisée par J.-C. Beacco et D. Lehman (1990) pour renvoyer à la fois au français instrumental, au français fonctionnel et au français de spécialité.

6 Elle est également employée par J.-M. Mangiante et Ch. Parpette $(2004: 16)$ pour désigner l'ensemble des publics "étudiant le français dans une perspective professionnelle ou universitaire ». Ainsi, l'expression «public spécifique» renvoie, actuellement (comme dans cet article), à l'ensemble des publics concernés par les LS, les LOS, les langues à visées professionnelles et les langues sur objectifs universitaires.

7 Les formations destinées aux PS ont effectivement des points communs qui justifient qu'elles soient regroupées dans un même champ de la didactique des langues. Ces publics sont, dans tous les cas, des adultes qui veulent acquérir ou perfectionner des compétences en langues étrangères pour réussir dans leurs études ou dans leurs activités professionnelles (J.-P. Cuq, 2003 : 109 ; J.-M. Mangiante et Ch. Parpette, 2004 : 16). Chaque formation se limite aux activités linguistiques concernées par une seule spécialité, par un seul métier, par une seule activité professionnelle, ou par un objectif spécifique.

8 Ceux qui assurent ces formations se préoccupent des "utilisations concrètes qu'il [l'apprenant] devra faire de la langue» qu'il apprend (C. Carras et al., 2007 : 22). Cela implique la sélection des contenus à enseigner en tenant compte des besoins langagiers présents et/ou futurs des apprenants. Ceux-ci sont déterminés en fonction des types de situations auxquels ces apprenants sont et seront confrontés, et des genres textuels (écrits et/ou oraux) qu'ils ont ou auront à comprendre et/ou à produire (J.-P. Cuq, 2003 :110 ; J.-M. Mangiante et Ch. Parpette, 2004 :17 ; J.-J. Richer, 2008).

9 Il convient de préciser qu'en dépit des différences qui singularisent chacune des formations dispensées aux PS, celles-ci ont en commun une ingénierie didactique très stricte par laquelle elles se distinguent des formations en langues « communes». Cette ingénierie est un processus en cinq étapes. Celles-ci sont pareillement expliquées et illustrées dans Le Français sur Objectif Spécifique : de l'analyse des besoins à l'élaboration d'un cours (Mangiante et Parpette, 2004 :7-9), pour des formations relevant des LOS, et dans Le Français sur objectif universitaire (Mangiante et Parpette, 2011 : 41-43), pour celles qui s'inscrivent dans les langues sur objectifs universitaires. En ce qui concerne les formations en LS, les mêmes étapes sont synthétisées sous le titre « La constitution d'un programme de langue de spécialité »:

C'est un processus en 5 étapes qui part d'une demande de formation, généralement institutionnelle, à partir de laquelle est élaborée une analyse des besoins. Cette analyse faite, le concepteur entreprend une collecte de données à partir desquelles 
il construira son programme de formation linguistique. Ces données authentiques doivent être ensuite analysées et traitées pour devenir des supports d'enseignement qui donneront naissance aux activités de classe. (Ch. Parpette, $2003: 1)$

10 Cette démarche se distingue par la part prépondérante qu'occupent les supports et les thématiques du domaine de spécialisation des apprenants. C'est d'ailleurs l'une des raisons pour lesquelles la « collecte de données » occupe une place primordiale dans le champ de la didactique des PS :

La collecte des données est probablement l'étape la plus spécifique à l'élaboration d'un programme de FOS. C'est en quelque sorte le centre de gravité de la démarche. D'une part, parce qu'elle confirme, complète, voire modifie largement l'analyse des besoins faite par le concepteur, laquelle reste hypothétique tant qu'elle n'est pas confirmée par le terrain. D'autre part, parce qu'elle fournit les informations et discours à partir desquels sera constitué le programme de formation linguistique. (Ch. Parpette et J.-M. Mangiante, 2004 : 02)

Qu'en est-il des formations en LA? Dans quelle mesure leur nature et leurs caractéristiques coïncideraient-elles avec celles des formations dispensées aux PS ?

Pour le savoir, nous devons, au préalable, cerner la nature et les particularités des formations en LA. Comme nous n'avons connaissance d'aucune définition satisfaisante de la notion de "langue appliquée », nous allons nous contenter d'analyser l'expression elle-même et divers programmes de formation placés sous sa bannière.

Nous interroger sur la nature et sur les caractéristiques des formations en LA exige que nous nous penchions sur le sens du déterminant «appliquée ». En général, celui-ci est opposé à "théorique ». C'est le cas dans l'expression "linguistique appliquée», qui désigne la partie des sciences du langage qui traite des relations entre celles-ci et les "différents domaines d'activité sociale", tels que l'enseignement et les politiques linguistiques (Cuq, 2003 :156). Il s'agit notamment d'une linguistique qui se soucie du transfert des résultats théoriques vers la pratique. C'est le cas des formations auxquelles nous nous référons dans cette étude.

Wolski-Quéré (2006 : 83), qui explique que l'objectif professionnel est au cœur même de l'esprit de ce type de formations, estime qu'opter pour les LA, c'est «mettre les langues au service de ... ». On peut lire dans la présentation d'une formation de master, intitulée "Langues de spécialité - Corpus - Traductologie », que "cette spécialité de Master se distingue des anciens diplômes LEA par son assise dans la recherche en linguistique appliquée (industries de la langue, langues de spécialité et traduction pragmatique) " (Université Paris Diderot, 2010). Une des formations de la Faculté des Langues Appliquées Commerce et Communication (FLACC) de l'Université Blaise Pascal (France) est intitulée «La formation LEA : une formation universitaire professionnalisée $»^{3}$. Il est précisé, dans la présentation de son programme, que celle-ci « repose sur les principes suivants : ouverture internationale, place des langues et professionnalisation ", et qu'elle vise à conduire les étudiants « à transformer des savoirs en savoir-faire et en compétences professionnelles, (...) à mettre en cuvre progressivement un projet professionnel " (Université Blaise-Pascal Clermont II, $2012: 4)$. Les concepteurs d'une licence en langues étrangères appliquées aux affaires de l'Université Lille 3 (France) précisent, quant à eux, que la formation en LA a pour objectif d'aider à l'insertion professionnelle :

Au cours de votre formation, vous élaborerez un projet professionnel (...) qui vous permettra d'acquérir une méthode de travail en vue de lier ce projet à votre formation. En troisième année, vous pourrez participer aux ateliers CV/Lettres de 
motivation, à des conférences (...) et autres événements pour une insertion professionnelle réussie. (Université Lille 3, 2013 : 2)

sont en rapport avec le domaine ciblé : «Langue orale: Thèmes de l'actualité, de la vie de
tous les jours ou du monde du travail », «Enseignements de la langue écrite visant à développer les qualités de rédaction dans un contexte professionnel », etc. (p.13).

18 De ce premier examen de la notion de LA et des programmes, nous retenons d'abord que les formations en LA, à l'instar de celles qui relevaient auparavant du champ de la didactique des PS, concernent des publics adultes (des étudiants ou des professionnels), qu'elles s'articulent autour d'un seul domaine de spécialisation (spécialité, discipline, métier, activité professionnelle, etc.), et qu'elles visent le développement de compétences qui permettent de réussir un projet universitaire et/ou professionnel. Nous relevons, ensuite, que les domaines de formation en LA sont très proches de ceux des PS (affaires, sciences, droit, etc.). Nous constatons, enfin, que les contenus discursifs et linguistiques des LA se distinguent nettement de ceux des langues « communes ».

19 L'enseignement/apprentissage des LA nécessite donc, de notre point de vue, une ingénierie didactique spécifique, comparable à celle des autres formations destinées aux PS. Cela implique que les publics des formations en LA sont à considérer comme des PS.

Il nous reste à étudier la position des LA par rapport aux LS et aux LOS et l'éventualité de leur constitution en sous-champ autonome de la didactique des langues.

\section{Langue(s) appliquée (s) : un nouveau sous-champ de la didactique des PS ?}

21 Plusieurs auteurs assimilent les LA aux LS. C'est le cas dans la définition retenue par l'Association du Français Appliqué : « "Français Appliqué" signifie français de spécialité ou français appliqué à des domaines tels que le monde des affaires, le tourisme, les sciences et la technologie, ainsi que les études interculturelles » (Association du français appliqué, 2012).

22 Il en est de même dans les écrits de F. Olmo Cazevieille $(2006,2008)$ qui parle de "français appliqué à une spécialité " (2008:199), et qui pense qu' " on enseigne les langues de spécialité puisque le français est appliqué aux différentes branches techniques » $(2006: 4)$. 

proposons de cerner cette problématique à travers le champ des langues appliquées (que nous illustrerons par l'exemple du FOS ${ }^{5}$ )», faisant ainsi du FOS un sous-champ des LA. entre les LA et les LOS :

Les apprenants auxquels nous allions nous adresser relevant d'un domaine bien spécifique et ayant à utiliser ce qui dans le domaine anglo-saxon est qualifié de English for Specific purposes (anglais à objectifs spécifiques ou langue appliquée), il nous apparaissait donc impératif de bien connaître le genre de la communication scientifique orale.

Pour la majorité des chercheurs, les formations en LA ne diffèrent pas de celles du souschamp des LS ou de celui des LOS. En effet, les formations en LA, en LS ou en LOS appartiennent au même champ, celui des PS, et réclament des ingénieries didactiques similaires (étude des besoins langagiers du public, collecte de données, didactisation de documents issus du domaine d'activité concerné par la formation, etc.). Nous pouvons ajouter que la plupart des spécialités, métiers ou activités professionnelles rattachés aux formations en LA (affaires, tourisme, droit, journalisme, économie, sport, ...) sont également rattachés aux formations en LS et en LOS. De plus, les situations, les genres textuels, les thématiques et les contenus grammaticaux et lexicaux qui prédominent dans les discours qui caractérisent la "spécialité » ou le métier occupent une place prépondérante ${ }^{6}$, aussi bien dans les LA que dans les autres formations destinées aux PS.

Pourtant, il convient de noter que certaines des caractéristiques des formations auxquelles nous nous intéressons dans cette étude sont spécifiques aux LA. Elles concernent essentiellement le nombre de langues impliquées, le profil des publics, les objectifs et les contenus.

Les formations en LA peuvent être monolingues, mais aussi bilingues ou plurilingues, tandis que les formations en LS et en LOS sont toujours monolingues. Parmi les intitulés en LA qui renvoient à des formations monolingues, citons, par exemple: «Langue appliquée aux sciences juridiques ", "Langue appliquée au contexte des affaires et du milieu du travail», "Langue appliquée aux sciences sociales et humaines", "Anglais appliqué à la recherche », « Anglais appliqué à l'histoire ».

Parmi les formations bilingues, voire plurilingues, citons : "Anglais et arabe appliqués au commerce", "Langues Appliquées aux Sciences et Techniques", "Langues Appliquées aux Sciences Juridiques », «Langues appliquées aux marchés des médias européens »7.

Les publics des formations en LS et en LOS sont des publics spécialisés dans un domaine autre que linguistique (Mourlhon-Dallies, 2008 : 73), et sont donc «non spécialistes » en langues (Mourlhon-Dallies, 2006). Ils sont médecins, économistes, juristes, physiciens, sportifs, politiciens, etc., ou des étudiants qui vont le devenir. Ils n'ont besoin, par conséquent, que d'un "complément» de formation linguistique (Bădulescu, 2005 : $522)^{8}$.

30 Ce n'est pas le cas de ceux des formations en LA, qui ont pour (principale) spécialité les métiers de langues, à l'exclusion des autres spécialités. Les spécialistes en langues, formés en LA, quelles que soient les activités ou les métiers qu'ils exercent, n'ont à s'occuper que des tâches qui nécessitent le recours aux langues dans un cadre spécialisé (accueil, secrétariats spécialisés, etc.). Les auteurs de l'une des présentations de formations en LA de l'université Paris Diderot, par exemple, expliquent que 
Les diplômes de Langues Appliquées de l'université Paris Diderot ne sont pas orientés vers le commerce, mais vers les langues de spécialité (pour les licences anglaisallemand et anglais-espagnol) et les échanges internationaux (pour les langues asiatiques). Ces formations préparent notamment à des métiers de la langue comme la traduction spécialisée, la documentation, la lexicologie, la terminologie ou la rédaction technique. Cette liste n'est cependant pas exhaustive: la pluridisciplinarité de la formation LEA permet l'accès à un éventail assez large de métiers, souvent grâce à une formation complémentaire appropriée. (Université Paris Diderot, 2012)

31 Par ailleurs, dans le cas des LS ou des LOS, la formation a pour objectif principal de permettre aux apprenants d'apprendre, dans une autre langue, des contenus qu'ils ont déjà acquis dans leur « spécialité ».

Les formations en LA prennent en charge, en plus des contenus relevant de la langue (des contenus discursifs, linguistiques, culturels, ...), des contenus de «spécialité » qui concernent les métiers ou les domaines d'activités ciblés. Les formations en LA n'interviennent pas en complément d'une (autre) formation de spécialité : elles restent, avant tout, des formations en langues, et ne peuvent concerner que les publics dont les spécialités relèvent essentiellement des langues. L'expression « les métiers de langues » est d'ailleurs assez fréquemment utilisée dans les documents et ouvrages portant sur les formations en LA.

33 C'est cette caractéristique qui justifie leur classement dans la catégorie des formations professionnalisantes.

34 A ce sujet, Ch. Soulas précise que les langues ne constituent pas des métiers : «Les langues, ce n'est pas un métier. Seule la combinaison des langues avec d'autres compétences, liées à l'administration ou à l'activité des entreprises, permet d'envisager des débouchés professionnels » (Soulas cité par Wolski-Quéré, 2006 : 83).

À titre d'exemple, dans le programme d'une licence en «Langues Appliquées, Commerce et Communication", de l'Université Blaise-Pascal (Clermont II 2012), figurent les unités suivantes : "Civilisation : Étude de certains aspects des sociétés : histoire, géographie, identités, population et représentation politique» (p. 10), et «Étude de certains aspects de la société britannique contemporaine: économie, relations sociales, systèmes éducatifs et de protection sociale, politique étrangère » (p. 14).

36 Il est également prévu, dans la même licence des enseignements consacrés à l'économie et au droit : Economie (10 CM et $10 \mathrm{TD})$, Droit (10 CM et $10 \mathrm{TD})$.

Wolski-Quéré (2006: 84) souligne, quant à lui, que dans une licence en LA, les contenus qui concernent le domaine d'application occupent jusqu'à $40 \%$ du volume horaire consacré à la formation.

38 Nous en déduisons que malgré leurs similitudes, les formations en LA se distinguent fondamentalement de celles en LS et en LOS, au point que nous nous autorisons à envisager la possibilité de leur constitution en sous-champ, autonome, de la didactique des formations des PS.

Le postulat de cette autonomie, par rapport aux LS et aux LOS, ouvre la voie à l'approfondissement de la recherche dans ce domaine. Le fait, par exemple, que les LA concernent généralement plus d'une langue devrait conduire les chercheurs à envisager de recourir aux démarches de l'enseignement bilingue et plurilingue. Ce sont ces démarches qui pourraient, par exemple, permettre de concevoir des activités de 
thème et version, de développer des compétences bilingues ou plurilingues et des compétences de médiation linguistique et culturelle.

Dans la mesure où les formations en LA prennent en charge les contenus de langue et les contenus de spécialité, le chercheur pourrait convoquer, outre la didactique des PS, les didactiques des disciplines concernées. En effet, même si aucune frontière rigide n'est établie entre les didactiques des langues et celles des autres disciplines, le développement de compétences disciplinaires ou de « spécialité » nécessite souvent des démarches didactiques nettement différentes de celles qui concernent l'enseignement/ apprentissage des langues.

Dans la présentation de l'ouvrage de Jonnaert et Laurin (2001), consacré aux didactiques des disciplines, il est noté que «penser la formation professionnelle à l'enseignement oblige à clarifier la contribution spécifique des didactiques des disciplines au "savoir-enseigner" ». Ainsi, l'examen de la notion elle-même de "langue appliquée » et des principales caractéristiques des formations qui prennent en charge ces langues nous a permis de conclure que les formations en LA sont à considérer comme des formations destinées à des PS. La confrontation des formations en LA avec celles en LS et en LOS révèle qu'elles appartiennent à des sous-champs différents et qu'il est nécessaire que les formations en LA constituent un nouveau sous-champ autonome de la didactique des PS. Nous nous appliquerons, dans des travaux ultérieurs en langues appliquées, à explorer cette hypothèse.

\section{BIBLIOGRAPHIE}

ASSOCIATION DE FRANÇAIS APPLIQUÉ, «Constitution en français », 2012. Disponible sur le site : http:// www.afa-irlande.ie/consfr.htm.

BADULESCU Sanda Marina, «Le renouveau des langues de spécialité dans l'année européenne de la citoyenneté par éducation ", The Proceedings of the "European integration-between tradition and modernity", Congress, V.1, 2005, pp. 520-532, [Disponible sur le site :

http://www.upm.ro/facultati_departamente/stiinte_litere/conferinte/situl_integrare _europeana/Lucrari/Badulescu.pdf

BEACCO Jean-Claude, LEHMANN Denis (Dirs.), Publics spécifiques et communications spécialisée, Le Français dans le Monde, Recherches \& Applications, Numéro spécial, 1990, Paris, Clé International/ FIPF.

CARRAS Catherine, TOLAS Jacqueline, KOHLER Patricia et SZILAGYI Élisabeth, Le français sur Objectifs Spécifiques et la classe de langue, Paris, Clé International, 2007.

CHATEAU Anne, «Spécificités des techniques d'enseignement par rapport aux publics, mythe ou réalité ? ", Recherches en didactique des langues, Actes du colloque Acedle juin 2005, Les Cahiers de l'Acedle, $\mathrm{n}^{\circ}$ 2, 2006, pp. 74-88. Disponible sur le site : http://acedle.org/IMG/pdf/ChateauA_cah2.pdf 
CUQ Jean-Pierre (Dir.), Dictionnaire de didactique du français langue étrangère et seconde, Paris, Clé International, 2003.

JONNAERT Philippe \& LAURIN Suzane, Les Didactiques des disciplines. Un débat contemporain, Montréal, Presses de l'Université du Québec, 2001.

MANGIANTE Jean-Marc, PARPETTE Chantal, Le Français sur Objectif Spécifique : de l'analyse des besoins à l'élaboration d'un cours, Paris, Hachette, 2004.

MANGIANTE Jean-Marc, PARPETTE Chantal, Le Français sur objectif universitaire, Grenoble, PUG, 2011.

MILED Mohamed, «Concevoir et mettre en œuvre un curriculum porteur de sens : Le cas du français sur objectifs spécifiques dans un contexte de langue seconde ", communication présentée au colloque internationale "Rapport au savoir : la problématique du sens", Sousse (Tunisie), 2007. Disponible sur le site : http://www.francparler.org/dossiers/flp2.htm

MOURLHON-DALLIES Florence, Enseigner une langue à des fins professionnelles, Paris, Didier, 2008.

OLMO CAZEVIEILLE Françoise, « Les unités simples et complexes du vocabulaire français de la zootechnie : perspectives linguistique, lexicographique et contrastive », Thèse de doctorat, 2006. Disponible sur le site $:$ http://tdx.cat/bitstream/handle/10803/9841/ lmo.pdf ?sequence =1 OLMO CAZEVIEILLE Françoise, «Quelle méthodologie adopter pour des élèves débutants en Français sur Objectifs spécifiques (FOS) ? ", in BERTRAND Olivier, SCHAFFNER Isabelle (dir.), Le Français de spécialité : enjeux culturels et linguistiques, Palaiseau, Éditions de l'École Polytechnique, 2008, pp. 197-206.

PARPETTE Chantal \& MANGIANTE Jean-Marc (2004) « Le Français sur Objectif Spécifique ou l'art de s'adapter ». Disponible sur le site : http://lesla.univ-lyon2.fr/sites/lesla/IMG/pdf/doc-592.pdf PARPETTE Chantal (2003) «Élaboration de programmes de français sur objectif spécifique et coopération internationale ». Disponible sur le site : http://lesla.univ-lyon2.fr/sites/lesla/IMG/ pdf/doc-188.pdf

RICHER Jean-Jacques, « Le FOS ou une didactique du langage et de l'action », Synergies Chine n ${ }^{\circ} 3$, 2008, pp. 15-30. Disponible sur le site : http://ressources-cla.univ-fcomte.fr/gerflint/Chine3/ richer2.pdf

UNIVERSITÉ BLAISE-PASCAL CLERMONT II, « LEA Langues étrangères appliquées. Programmes et règlements 2012-2013. $1^{\text {ère }}$ année de licence ", 2012. Disponible sur le site : http://www.lacc.univbpclermont.fr/IMG/pdf/Livret_LEA1.pdf

UNIVERSITÉ LILLE 3, « Langues étrangères appliquées aux affaires. Licence 1 - Licence 2 - Licence 3 », 2013. Disponible sur le site : http://documents.univ-lille3.fr/files/pub/www/formations/ licences/licence_lea.pdf

UNIVERSITÉ PARIS DIDEROT, « La filière LEA : Langues Étrangères Appliquées », 2012. Disponible sur le site : http://www.eila.univ-paris-diderot.fr/enseignement/lea/index

UNIVERSITÉ PARIS DIDEROT, «M2 Langues Appliquées, spécialité Langues de Spécialité, Corpus et Traductologie Recherche ». Disponible sur le site : www.eila.univ-paris-diderot.fr/enseignement/ lea/master/recherche WOLSKI-QUÉRÉ Murielle, Bien choisir sa licence : Spécial LMD, Paris, Éditions l'étudiant, 2006. 


\section{NOTES}

1. Nous utilisons cette expression générique pour désigner l'ensemble des publics qu'on oppose généralement à celui des langues dites générales ou communes.

2. Il est à préciser que la désignation « langue appliquée » n'est pas citée dans les répertoires des appellations des formations dispensées aux PS, y compris dans les plus récents proposés dans les ouvrages de Carras et al. (2007:17-18) et de Mourlhon-Dallies (2008:13-14).

3. LEA : Langues Étrangères Appliquées.

4. Les formations indiquées à travers ces intitulés et ceux que nous donnons entre guillemets tout au long de cet article sont proposées et assurées par des universités ou par des centres de formation en langues.

5. FOS : Français sur Objectif(s) Spécifique(s)

6. Cela résulte, comme nous l'expliquions plus haut, d'une part de la nécessité de viser l'enseignement/ apprentissage des genres textuels qui caractérisent la spécialité, le métier ou l'activité professionnelle des apprenants (Richer, 2008), et, d'autre part, du fait que les supports écrits et oraux utilisés pour concevoir les activités et les tâches sont le plus souvent ceux obtenus dans le cadre de l'analyse des besoins et de la collecte de données (Mangiante et Parpette, 2004).

7. Ces intitulés de formations monolingues, bilingues ou plurilingues dénotent de la diversité des formations qui prennent en charge les LA, et de la grande similitude entre les domaines et les métiers auxquels renvoient ces formations et ceux des formations dont bénéficient généralement les PS.

8. En traitant des publics concernés par les langues sur objectif(s) spécifique(s), Bădulescu déclare qu'il s'agit de publics "qui ne sont pas d'étudiants de langue. S'ils étudient les langues de spécialité c'est que cette formation leur est nécessaire en complément d'autre chose ».

\section{RÉSUMÉS}

Dans cette étude, nous comptons démontrer que les langues appliquées sont à même de constituer un sous-champ à part entière de la didactique des langues. Or, jusqu'à présent, les langues appliquées sont souvent assimilées à des langues de spécialité ou des langues sur objectifs spécifiques. Pourtant, la notion même de «langue appliquée » et l'examen du contenu des formations initiées pour leur enseignement/apprentissage montrent qu'il s'agit de formations spécifiques destinées à des publics spécifiques. En comparant les principales formations en langues appliquées et les formations en langues de spécialité ou sur objectifs spécifiques, nous concluons ici même à l'émergence d'un nouveau sous-champ de la didactique et à la nécessité de l'appréhender dans son autonomie.

In this study we intend to demonstrate that applied languages are able to constitute a complete subfield of language didactics. However, until now, applied languages are often treated as Languages Specialties (LS) or as Languages for Specific Purposes (LSP). Yet, the notion of "applied language(s) to..." and the examination of the course features aiming at their teaching/learning shows that we are dealing with specific training for specific audiences. The comparison of the main features of language training courses with those applied in specialized languages or languages for specific purposes leads to conclude, here, that we are witnessing the emergence of a new sub-field of didactics, which must necessarily be considered as independent. 
INDEX

Mots-clés : public spécifique, langue appliquée, langue de spécialité, langue sur objectif spécifique

Keywords : specific audience, applied language, language specialties, language for specific purpose

\section{AUTEUR}

\section{M'HAND AMMOUDEN}

Université A. Mira - LAILEMM - Bejaia 